\title{
Lewy Body Dementia as an Underlying Etiology for Posterior Cortical Atrophy Initially Presented with Visual Agnosia and Alexia: A Case Report
}

Akiko Ishiwata*, Chikako Nito and Kazumi Kimura

Departments of Neurological Science, Graduate School of Medicine, Nippon Medical School, 1-1-5 Sendagi, Bunkyo-ku, Tokyo, 113-8603, Japan

*Corresponding author: Akiko Ishiwata, Department of Neurological Science, Graduate School of Medicine, Nippon Medical School, 1-1-5 Sendagi, Bunkyo-Ku, Tokyo, 113-8603, Japan, Tel: +81-3-3822-2131; E-mail: akiko081@nms.ac.jp

Received date: July 01, 2015; Accepted date: August 01, 2015; Published date: August 06, 2015

Copyright: (C2015 Ishiwata A et al. This is an open-access article distributed under the terms of the Creative Commons Attribution License, which permits unrestricted use, distribution, and reproduction in any medium, provided the original author and source are credited.

\begin{abstract}
Posterior cortical atrophy (PCA) is a neurodegenerative disorder with cerebral atrophy in the parietal, occipital, or occipitotemporal cortices, and is characterized by visuospatial and visuoperceptual impairments. The majority of the disorder is compatible pathologically with Alzheimer's disease (AD). However, many patients show no memory loss until late stages of the disease. We encountered a case where a combination of several imaging modalities, in conjunction with symptoms and neuropsychological exams, led not only to a diagnosis of PCA but also identified its underlying etiology. A 62-year-old Japanese man presented to ophthalmology with visual impairment and without abnormalities in his visual acuity or visual fields. Alexia was identified, particularly pertinent to reading Kanji, at the neurology clinic. His initial Mini-Mental State Examination (MMSE) revealed a score of 27/30 points with delayed recall $(-2)$ and constructional apraxia $(-1)$. The score dropped to 25 points six months later. Brain MRI demonstrated occipital atrophy. Brain single photon emission computed tomography (SPECT) showed hypoperfusion in the medial and lateral occipital lobes. The myocardial meta-iodobenzylguanidine (MIBG) scintigraphy showed no evidence of myocardial postganglionic sympathetic dysfunction. Posterior cortical atrophy (PCA) was diagnosed in conjunction with the symptoms, including alexia and visual impairment. He developed symmetric parkinsonism one year later. Lewy body dementia (DLB) was thus considered as the underlying etiology for the PCA. Additionally, alexia pertinent to reading Kanji has not been reported before. We herein report this rare case.
\end{abstract}

Keywords Posterior cortical atrophy; Lewy body dementia; Single photon emission computed tomography; Myocardial metaiodobenzylguanidine scintigraphy; Visual agnosia; Constructional apraxia; Alexia

\section{Introduction}

Posterior cortical atrophy (PCA) is a neurodegenerative disorder with cerebral atrophy in the parietal, occipital, or occipitotemporal cortices, and is characterized by visuospatial and visuoperceptual impairments. The majority of the disorder is compatible pathologically with Alzheimer's disease (AD). However, many patients show no memory loss until late stages of the disorder [1]. We encountered a case where a combination of several imaging modalities in conjunction with the symptoms and neuropsychological exams not only made the diagnosis of PCA but also identified its underlying etiology. In addition, we discuss symptoms of alexia related to the specific Japanese language system.

\section{Case report}

A 62-year-old right-handed retired Japanese man with 12-years of education background complained of progressive visual impairment for a period of six months. An ophthalmology clinic found no any abnormality in his visual acuity (right 20/50 and left 20/40) or visual fields. He was thus referred to a neurology clinic. For the neurological examination, upon motor, sensory, coordination and cranial nerve system, no relevant findings were elicited. Furthermore, constructional dyspraxia and apraxia, characterized by PCA, were present. In particular, the patient could not read some Kanji. On the other hand, simultanagnosia, visual field deficit, Gerstmann syndrome, which may be seen in PCA, were not present.

His past medical history, family history, and lab tests were unremarkable.

MMSE: 27/30 (delayed recall: -2 points and construction apraxia: -1 point).

Brain MRI demonstrated severe atrophy in the bilateral occipital lobes on FLAIR. Atrophy was also present in the parietal lobe (Figure $1)$.

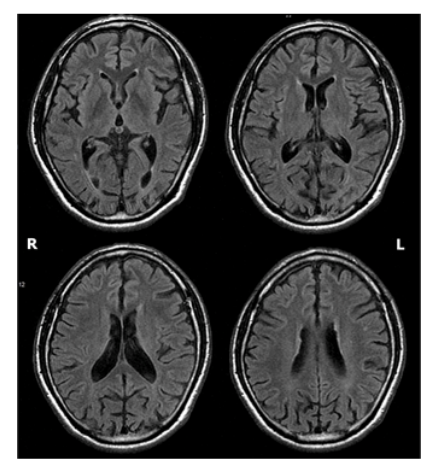

Figure 1: MRI FLAIR image; Severe bilateral occipital atrophy is visualized. There is also mild atrophy in the superior aspect of the parietal lobes. 
${ }^{123}$ I-IMP SPECT showed low perfusion in the bilateral occipital cortices, more prominent in the medial aspects (Figure 2).

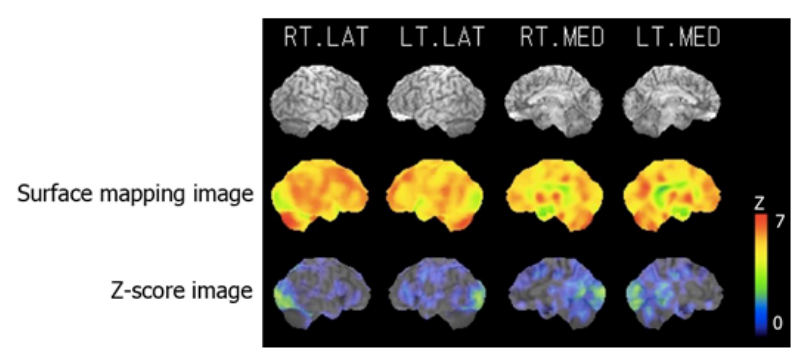

Figure 2: ${ }^{123} \mathrm{I}-\mathrm{MIBG}$ cardiac scintigraphy. In the early phase, the ratio of I-123 MIBG uptake between heart and the upper mediastinum $(\mathrm{H} / \mathrm{M})$ was 2.801 . In the delayed phase, the ratio was 3.150. There is no scintigraphic evidence of myocardial postganglionic sympathetic dysfunction.

Cardiac MIBG scintigraphy demonstrated a heart-to-mediastinum ratio $(\mathrm{H} / \mathrm{M})$ of 2.801 in the early image and 3.150 in the delayed image.

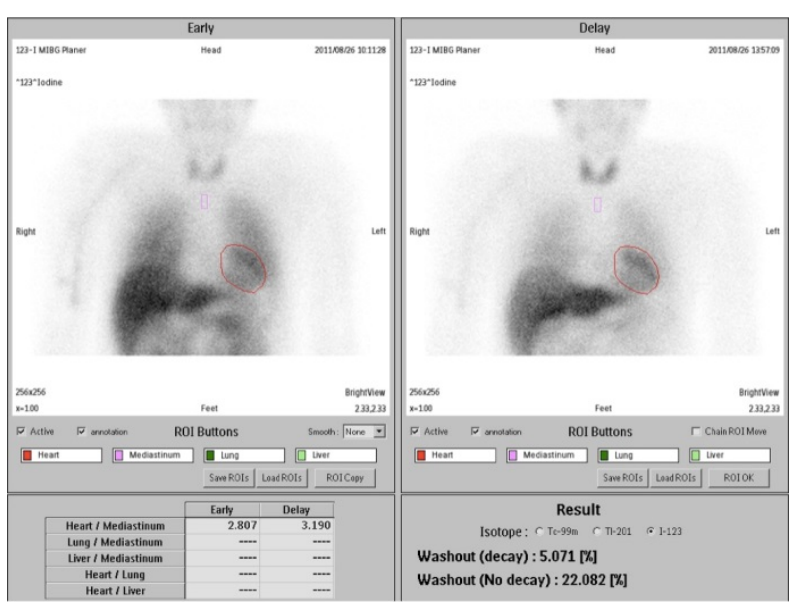

Figure 3: SPECT, surface mapping, and Z-score images. A fully automated three-dimensional stereotactic surface projection technique (3D-SSP) was used to analyze SPECT images. In brief, each SPECT image set was co-registered and warped to the common stereotactic coordinate system. Subsequently, gray matter perfusion was extracted to predefine surface pixels on a pixel-bypixel basis. The extent and significance of perfusion reduction in each subject and group were assessed on a Z-score map. The Zscore was defined as (normal mean - patient value)/(normal SD). $\mathrm{Z}$-score images were normalized by cerebellum perfusion. Color coding represents Z-score compared with age-matched normal controls. Red represents more significant perfusion reduction. There is low perfusion in bilateral occipital cortices, in particular in the medial aspects seen on surface mapping image (middle row) and Z-score image (lower row). Note: Right lateral (RT.LAT), left lateral (LT.LAT), right medial (RT.MED) and left medial (LT.MED) views of brain.
The patient jumped from his 6-floor apartment due to the stress of visual impairment one year after diagnoses (Figure 3). He was transported to an emergency department with multiple injuries (right pneumothorax, lung contusion, pelvic injury and sacral fracture). After undergoing rehabilitation in another hospital for two months he was able to walk with a cane. However, he could not walk without assistance due to the visual impairment. He demonstrated parkinsonism symptoms such as small steppage gait, postural disturbance, and rigidity. Seven months from the initial test, his MMSE score dropped to 25 points with delayed recall (-3), serial 7 (-1) and constructional apraxia (-1). He was identified as having tremors, rigidity of upper and lower extremities, stooped posture, and retropulsion at our clinic.

\section{Discussion}

This patient presented with visual agnosia, constructional apraxia, and alexia and subsequently developed extrapyramidal symptoms. Mild cognitive disorder is possible considering his age and educational background in evaluating cognitive function by MMSE [2].

On morphologic imaging, there is occipital atrophy. On SPECT, low perfusion is displayed in the medial and lateral occipital lobes, a characteristic feature of DLB. When this infrequent feature is present, DLB should be considered in the differential diagnoses. Myocardial postganglionic sympathetic dysfunction is another characteristic feature of DLB identified on myocardial MIBG scintigraphy [3]. Because our patient lacks this feature, he is considered 'possible DLB' per the DLB guideline [4]. In addition, our patient presented initially with visual agnosia and alexia, which could not be explained by DLB, but could be explained by PCA.

Our patient had PCA findings: a clinical course that matched PCA (slow progression and onset of visual impairment), symptoms (visual agnosia and constructional apraxia present without optic ataxia or motor paralysis or sensory disturbance), and by exam (predominant posterior atrophy on MRI) as proposed by Benson et al in 1988 [5]. Therefore, PCA was diagnosed. Our patient developed parkinsonism in the course of the disease. According to Snowden et al., PCA has the same incidence in parkinsonism (41\%), myoclonus (24\%), and grasp reflex (26\%) as it does in AD. In addition, when PCA is associated with symmetric parkinsonism, DLB is considered as the underlying etiology. When there is asymmetric myoclonus and dystonia, corticobasal syndrome (CBS) should be considered as the underlying etiology [6]. In our patient, DLB is the possible underlying etiology. Patients should thus be routinely followed with symptomology and imaging studies.

One article reported nine patients with autopsy had proven PCA. Among the nine patients, two patients displayed AD and two CBS [7]. Another article reported on 27 autopsied patients. Among them, 13 displayed $\mathrm{AD}$, one $\mathrm{AD}$ plus Parkinson's disease, two AD-Lewy body variant, two CBS, and two Prion disease [8]. Thus, although AD is more prevalent, non-AD diseases should also be considered. Concerning therapy of PCA, there has been no definite established protocol. However, there are case reports stating that PCA or a visual form of Alzheimer's disease is responsive to cholinesterase inhibitor $[9,10]$. The responsiveness to cholinesterase inhibitor may be due to the etiologies of the PCA in both reports. We speculate that cholinesterase inhibitor may be an effective therapy for PCA in those patients with Alzheimer's disease. Response to the treatment of the underlying etiology has been reported in some patients. Therefore, 
Citation: Ishiwata A, Nito C, Kimura K (2015) Lewy Body Dementia as an Underlying Etiology for Posterior Cortical Atrophy Initially Presented with Visual Agnosia and Alexia: A Case Report. J Neurol Neurophysiol 6: 305. doi:10.4172/2155-9562.1000305

Page 3 of 3

efforts should be made to use multiple imaging modalities to identify the underlying etiology. In comparison with $\mathrm{AD}$, patients with PCA have greater insights into their illness and are more susceptible to developing depression [11]. For this reason, antidepressive therapy would have been considered necessary for patients with PCA such as ours.

Furthermore, in our case, the patient had alexia characterized by an inability to read Kanji. Japanese is a unique language system, which is composed of phonograms like Kana and logograms like Kanji. Each Kana has one character and one corresponding phonation without exception. Each Kanji, however, may have several phonations. In Japan, it is still a subject of debate whether the responsible pathology for Kana alexia and Kanji alexia may be different. On the other hand, two distinct types of alexia have been reported in viewing the English language [12]. The differences in the responsible lesions may be characterized in the future. Nevertheless, alexia has been found to relate to lesions in occipitotemporal gyrus (Brodmann area 37) and posterior fusiform gyrus and inferior occipital gyrus (Brodmann area 17/18) [13]. The location of cerebral atrophy in our patient is consistent with these areas.

\section{Take home message:}

1. Degenerative cognitive disorders rarely show occipital atrophy. Posterior cortical atrophy should be included in the differential diagnoses in cases where there is occipital atrophy.

2. Alexia is key to early identification of cognitive disorders.

3. PCA has different pathologic processes. Since it has been reported that it is possible to treat the underlying etiology, a combination of morphologic and functional evaluations should be performed to identify the underlying etiology.

4. When PCA is associated with symmetric parkinsonism, DLB should be considered to be the underlying etiologSr. When PCA is associated with asymmetric myoclonus and dystonia, CBS should be considered to be the underlying etiolory.

\section{Acknowledgement}

The authors thank: Dr. Baocheng Chu and Mr. Zach Miller for English revisions.

\section{References}

1. Mendez MF, Shapira JS, Clark DG (2007) "Apperceptive" alexia in posterior cortical atrophy. Cortex 43: 264-270.

2. Crum RM, Anthony JC, Bassett SS, Folstein MF (1993) Population-based norms for the Mini-Mental State Examination by age and educational level. JAMA 269: 2386-2391.

3. Nagayama H, Hamamoto M, Ueda M, Nagashima J, Katayama Y (2005) Reliability of MIBG myocardial scintigraphy in the diagnosis of Parkinson's disease. J Neurol Neurosurg Psychiatry 76: 249-251.

4. McKeith IG, Dickson DW, Lowe J, Emre M, O'Brien JT, et al. (2005) Diagnosis and management of dementia with Lewy bodies: third report of the DLB Consortium. Neurology 65: 1863-1872.

5. Benson DF, Davis RJ, Snyder BD (1988) Posterior cortical atrophy. Arch Neurol 45: 789-793.

6. Snowden JS, Stopford CL, Julien CL, Thompson JC, Davidson Y, et al. (2007) Cognitive phenotypes in Alzheimer's disease and genetic risk. Cortex 43: 835-845.

7. Renner JA, Burns JM, Hou CE, McKeel DW Jr, Storandt M, et al. (2004) Progressive posterior cortical dysfunction: a clinicopathologic series. Neurology 63: 1175-1180.

8. Tang-Wai DF, Graff-Radford NR, Boeve BF, Dickson DW, Parisi JE, et al. (2004) Clinical, genetic, and neuropathologic characteristics of posterior cortical atrophy. Neurology 63: 1168-1174.

9. Kim E, Lee Y, Lee J, Han SH (2005) A case with cholinesterase inhibitor responsive asymmetric posterior cortical atrophy. Clin Neurol Neurosurg 108: 97-101.

10. Warren S, Hier DB, Pavel D (1998) Visual form of Alzheimer's disease and its response to anticholinesterase therapy. J Neuroimaging 8: $249-252$

11. Mendez MF, Ghajarania M, Perryman KM (2002) Posterior cortical atrophy: clinical characteristics and differences compared to Alzheimer's disease. Dement Geriatr Cogn Disord 14: 33-40.

12. Rosazza C, Appollonio I, Isella V, Shallice T (2007) Qualitatively different forms of pure alexia. Cogn Neuropsychol 24: 393-418.

13. Sakurai Y (2004) Varieties of alexia from fusiform, posterior inferior temporal and posterior occipital gyrus lesions. Behav Neurol 15: 35-50. 